\title{
The study of Sports Injuries treatment with Korean Medicine : A survey of World Martial Arts Masterships' athletes and officials
}

\author{
Jaesung Lee ${ }^{1}$, Jonghan Park ${ }^{2}$, Jangmi Park ${ }^{1}$, Chamkyul Lee ${ }^{1}$, Suhyeon Jeong ${ }^{2}$, \\ Kyunghwan Kong ${ }^{3}$, Eunyong Lee ${ }^{1^{*}}$ \\ ${ }^{1}$ Department of Acupuncture \& Moxibustion Medicine, Semyung University Chungju Hospital of Korean Medicine \\ ${ }^{2}$ Department of Rehabilitation Medicine of Korean Medicine, Semyung University Chungju Hospital of Korean \\ Medicine \\ ${ }^{3}$ Department of Korean Internal Medicine, Semyung University Chungju Hospital of Korean Medicine
}

Objectives: The objective of this study was to report the general characteristics, satisfaction, and changes before and after treatment to foreign athletes and officials who were received Korean Medicine at the 2019 Chungju World Martial Arts Masterships Korean Medical Clinic.

Methods: We investigated age, gender, nationality, type of sports, and, onset through medical records. Besides, we developed the patient satisfaction survey to determine how satisfied the patients were with the treatment they received. The survey included type of injury, location of the injury, cause of injury, Numeric Rating Scale (NRS) before and after treatment, and treatment satisfaction.

Result: This study showed that knee and lower leg injuries (S80-S89) were the most frequent injuries in the Korean Medicine(KM) clinic of the 2019 Chungju World Martial Arts Masterships. We provided KM treatments including acupuncture, cupping therapy, Chuna manual therapy, herbal medicine, dressing, and taping. Acupuncture was the most frequently performed and the most satisfactory treatment. The average NRS after treatment significantly decreased, suggesting that KM is effective in controlling pain in sports injuries. The overall satisfaction was also high with $\mathrm{KM}$ treatments.

$\overline{\text { Key Words }}$ : Korean Medicine, Sports Medicine, athletes, martial arts

\section{Introduction}

Korean Medicine(KM) has been developed through several studies, but studies regarding treatment of sports injuries with $\mathrm{KM}$ are relatively insufficient. Although specific treatment according to the type of sports injury in $\mathrm{KM}$ has not been confirmed yet, several studies have confirmed that acupuncture, cupping therapy, taping, and Chuna manual therapy provide mechanical stimulation to the skeletal and connective tissues and, thus, have therapeutic effects. KM can be applied to manage pain as a result of sports injury and to improve exercise performance. [1]

\footnotetext{
- Received : 31 October 2019

- Revised : 20 November 2019

- Accepted : 21 November 2019

- Correspondence to : Eunyong Lee

Department of Acupuncture \& Moxibustion Medicine, Semyung University Chungju Hospital of Korean Medicine

Semyung University Chungju Hospital of Korean Medicine, 63, Sangbang 4-gil, Chungju-si, Chungbuk-do, 27429,

Republic of Korea

Tel: +82-10-6269-0762, E-mail: acupley@naver.com
} 
Since the operation of the Acupuncture Department at the Athletes' Village Hospital in the 1988 Seoul Olympics, the 2014 Incheon Asian Paralympics, the 2016 Cheongju World Martial Arts Masterships, the 2018 Pyeongchang Winter Paralympics, and the 2019 Gwangju World Swimming Championships, studies about sports $\mathrm{KM}$ in operating the Korean medical clinics have been conducted. [2-6]

The 2019 Chungju World Martial Arts Masterships was a global martial arts competitions organized by the World Martial Arts Masterships Committee. It was held between August 30 and September 6 in Chungju, Korea and involved 20 sports with a total participation of 3,000 athletes from 100 countries. Korean medical clinic provided KM therapy (acupuncture, cupping therapy, Chuna manual therapy, herbal medicine, dressing, and taping) to taekwondo, judo, hakido, wushu, jujitsu, and ssireum athletes and the official staff from August 30 to September 5.

The authors reported the general characteristics, satisfaction, and Numeric Rating Scale (NRS) changes of foreign athletes and official staff treated at Korean medical clinics who were not familiar with KM at the 2019 Chungju World Martial Arts Masterships.

\section{Materials and Methods}

\section{Subject}

The subjects were 68 cases of foreign medical treatment from August 30 to September 5 at the 2019 Chungju World Martial Arts Masterships Korean Medical Clinic.

\section{Method}

We investigated age, gender, nationality, type of sports, and, onset through 68 medical records [Appendix 1]. Besides, we developed the patient satisfaction survey [Appendix 2] to determine how satisfied the patients were with the treatment they received. The survey included type of injury, location of the injury, cause of injury, NRS before and after treatment, and treatment satisfaction. At the end of the treatment, the patient anonymously made a survey themselves, and we obtained 49 surveys.

\section{Statistical analyses}

Statistical analyses were performed with IBM SPSS program Ver. 22.0K (IBM Corp., USA). All measurements are expressed as frequency (percentage). Statistical significance level is $\mathrm{p}<0.05$.

\section{Result}

\section{General Characteristic}

The results were investigated 42 cases $(61.7 \%)$ of males and 26 cases(38.2\%) of female with an average age of $27.88( \pm 12.19)$ among 68 cases [Table 1]. Nationalities of patients were investigated 18 cases $(26.5 \%)$ of Philippines, 10 cases $(14.7 \%)$ of Iran, 9 cases (13.2\%) of Colombia, 4 cases (5.9\%) of Kazakhstan, 3 cases (4.4\%) of Germany, 3 cases (4.4\%) of US, 3 cases (4.4\%) of Canada, 3 cases $(4.4 \%)$ of Thailand, 2 cases $(2.9 \%)$ of Russia, 2 cases (2.9\%) of Morocco, 2 cases (2.9\%) of Singapore, 2 cases $(2.9 \%)$ of Italy. There also was one case $(1.5 \%)$ each of South Africa, Mongolia, Belgium, Uzbekistan, India, Poland, and France [Figure 1]. Sports of patients were 29 cases $(43.6 \%)$ of taekwondo, followed by 
Table 1. Gender and age

\begin{tabular}{lccc}
\hline & Male & Female & Total \\
\hline Number & 42 & 26 & 68 \\
Age(mean \pm standard deviation) & $28.93 \pm 13.26$ & $26.19 \pm 10.25$ & $27.88 \pm 12.19$ \\
\hline
\end{tabular}

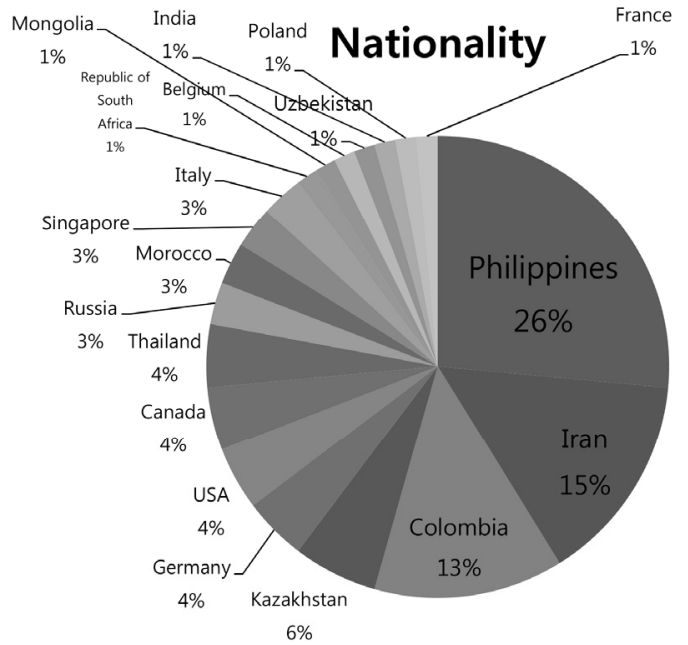

Fig. 1. Nationalities

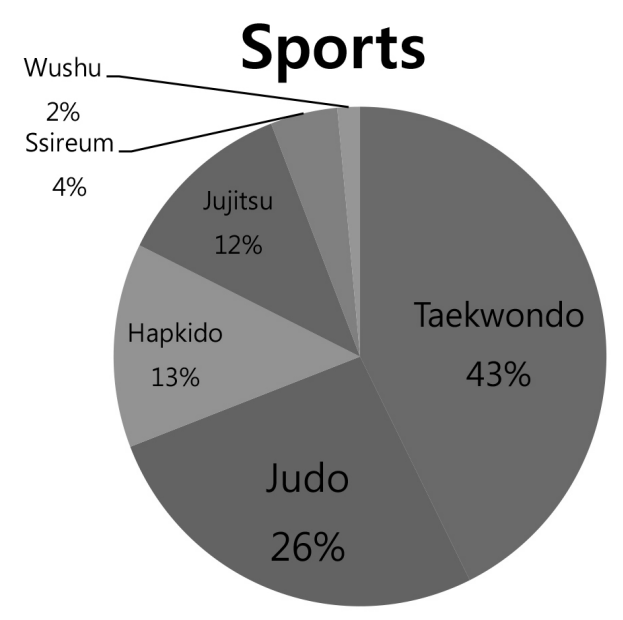

Fig. 2. Sports
18 cases $(26.5 \%)$ of judo, 9 cases $(13.2 \%)$ of hapkido, 8 cases $(11.8 \%)$ of jiu-jitsu, 3 cases $(4.4 \%)$ of ssireum, and 1 case $(1.5 \%)$ of wushu [Figure 2].

\section{Diagnosis}

Diagnosis was classified according to ICD-10. The most common diagnosis was 18 cases (26.5\%) of knee and lower leg injuries (S80-S89), followed by 10 cases (14.7\%) of neck injuries (S10-19), 9 cases (13.2\%) of abdomen, lower back, lumbar spine and pelvis injuries (S30-S39), 8 cases (11.8\%) of ankle and foot injuries (S90-S99), 7 cases (10.3\%) of shoulder and upper arm (S40-S49), 5 cases (7.4\%) of hip and thigh injuries (S70-S79), 4 cases (5.9\%) of common cold (J00), 2 cases $(2.9 \%)$ of head injuries
(S00-S09), 2 cases $(2.9 \%)$ of wrist and hand injuries (S60-S69), 2 cases $(2.9 \%)$ of thorax injuries (S20-S29), 1 case (1.5\%) of elbow and forearm injuries (S50-S59) [Figure 3].

\section{Numeric Rating Scale}

We investigated NRS to evaluate the degree of pain before and after KM treatment through 49 surveys we obtained. The mean NRS before treatment was $5.88( \pm 1.45)$ and the mean NRS after treatment decreased to $3.07( \pm 1.63)$, and p-value was 0.046 showing statistically significant decrease [Figure 4]. NRS difference between before and after treatment according to the type of injury was $3.01( \pm 1.59)$ for muscle injury, 3.00 ( \pm 3.00$)$ for others, $2.43( \pm 2.44)$ for joint (ligament) injury. There was no difference in NRS for bone 


\section{Diagnosis}

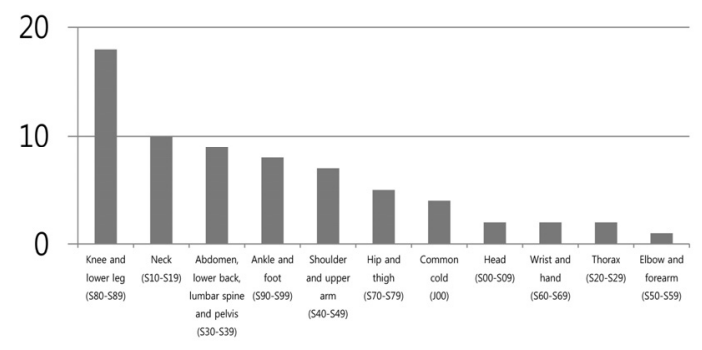

Fig. 3. Diagnosis

NRS

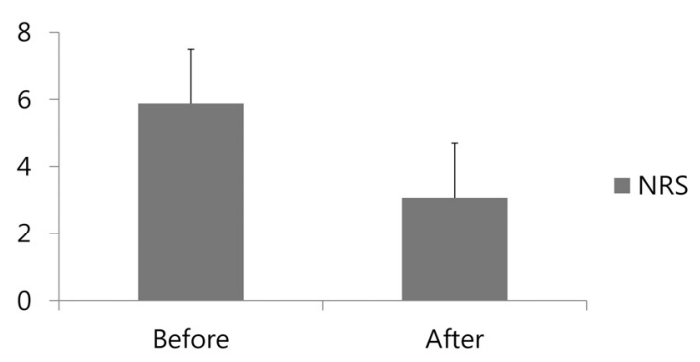

Fig. 4. NRS before and after treatment $(p<0.05)$

injury or laceration, but difference between types of injury was not statistically significant. NRS difference between before and after treatment according to cause of injury was $3.11( \pm 1.47)$ for aggravation or exacerbation of an existing injury, $3.00( \pm 4.24)$ for Illness, $2.82( \pm 1.74)$ for new injury, $2.40( \pm 2.70)$ for others, $2.33( \pm 2.07)$ for recurrence of previous injury that had fully resolved, but difference between causes of injury was not statistically significant.

\section{Treatment satisfaction}

We investigated received treatment and the most satisfactory treatment through 49 surveys we obtained. The patients who answer the survey received 32 cases of acupuncture, 27 cases of cupping, 18 cases of taping, 12 cases of chuna

\section{The most satisfactory treatment}

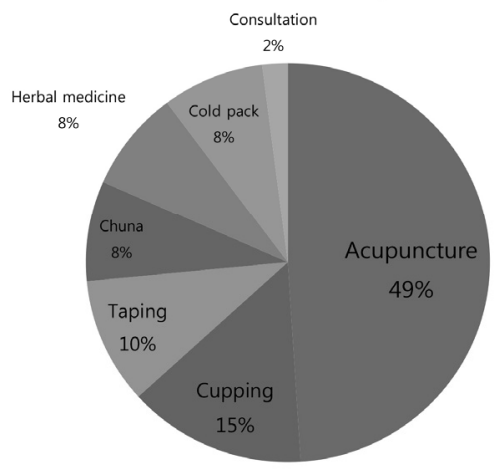

Fig. 5. The most satisfactory treatment

manual therapy, 5 cases of herbal medicines, 9 cases of ice pack, and 2 cases of consultation, and the most satisfactory treatment was acupuncture (24 cases, 49.0\%), followed by cupping (7 cases, $14.3 \%$ ), taping (5 cases, 10.2\%), chuna manual therapy (4 cases, $8.2 \%$ ), herbal medicine (4 cases, $8.2 \%$ ), ice pack ( 4 cases, $8.2 \%$ ), and consultation (1 case, 2.0\%) [Figure 5]

\section{Satisfaction}

According to 49 surveys on overall satisfaction with KM treatment, 37 cases $(75.5 \%)$ were highly satisfied, 10 cases (20.4\%) satisfied, and 2 cases (4.1\%) partially satisfied. Most patients were satisfied with KM treatment [Figure 6].

\section{Discussion}

As the sports population for health increases, the need for sports injury also increases. Definitions of sports medicine vary, Ryan defines sports medicine as the medical management of athletes involved in, special sports, exercise therapy, and exercise for the prevention of chronic degenerative diseases. [7] Sports injuries can be 


\section{Satisfaction}

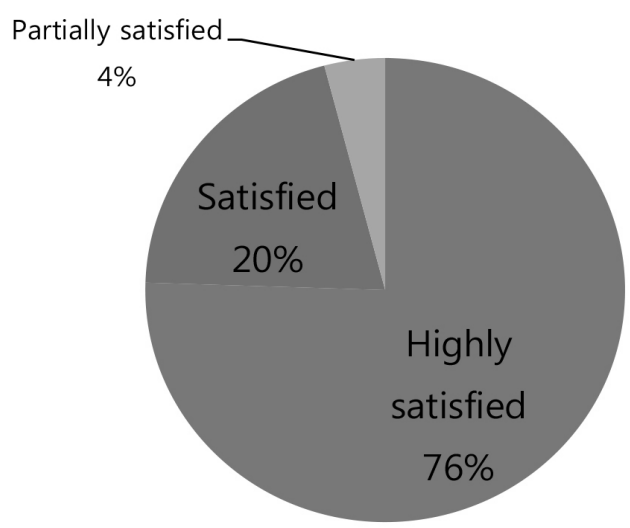

Fig. 6. Satisfaction

divided into acute and chronic injuries. Acute injuries include fractures, dislocations, and ligaments and muscle ruptures, and chronic injuries include tendonitis, fatigue fractures, chondromalacia, and chronic compartment syndrome. Since sports injuries are often caused by repeated movements involved in each sport, it is important to understand sports movements and their possible damage mechanisms [8]. Sports injuries usually occur in the knee, lumbar spine, shoulder, and ankle, and contusion, tendonitis, and sprain are more common than fracture or dislocation. [7] Most patients were diagnosed with knee and lower leg injuries in this study.

Acupuncture, cupping therapy, taping, Chuna manual therapy, and physical therapy as part of KM treatments can be applied to sports medicine, and it has been reported that they have therapeutic effects in skeletal-muscular disorders as confirmed by several clinical experiences and studies. Sports injuries are similar to Sang-guen(傷筋) in KM. They are painful, and lead to functional disorder caused by trauma, and are treated with acupuncture, moxibustion, or herbal medicine depending on the patterns identified in KM. [9]

With the advancement of medicine and medical science, acupuncture has been studied. Particularly, several studies have been conducted on the analgesic mechanisms of acupuncture, and acupuncture has been considered a major complementary and alternative medicine techniques. Acupuncture is widely used in sports injuries for elite athletes, because it rapidly acts on the injured site and is easily applicable [10, 11]. It is similar that most patients who visited the Korean Medicine Clinic chose acupuncture as the most satisfactory treatment. Additionally, despite the absence of injury, acupuncture is effective in improving exercise ability and recovery after exercise because acupuncture increases power output, and stabilizes blood pressure, heart rate, and oxygen consumption before and after exercise [12-13]. Hence, acupuncture has an important role in pain control and improvement of sports performance in sports medicine.

Cupping is based on the Seo-geun-hwal-lak(舒 筋活絡) effect, which includes blood circulation stimulation, and causing the muscles and joints to relax by removing blood stasis. [9] Hence, this therapy can be applied to muscle and joint diseases and is used to reduce pain and improve sports performance. Studies regarding cupping therapy in sports medicine are insufficient, but the use of cupping has increased recently in elite athletes worldwide. [14]. The study showed that cupping was the second most used and satisfactory treatment.

Chuna therapy is a KM therapy aimed at managing pain and correcting imbalance. Sports involve the repetition similar movements depending on the sport, so it is easy to cause the 
load in a particular area and imbalance in human body. [8] Chuna therapy seems to help restore sports damage from this reason. Taping therapy is called Cheop-dae(貼帶) in KM, and it has been developed and used based on meridians and acupuncture points, which are unique theories of $\mathrm{KM}$, as well as joint fixation and muscle strengthening. [7] It does not show faster effects than other KM treatments in managing pain, but it can be used to prevent sports injury in sports medicine. Herbal medicine was infrequently used in the KM clinic, because athletes and coaches were cautious about unintended anti-doping rule violations. There are a few studies about herbal medicine related anti-doping rules, but more studies are required to establish the appropriate herbal medicine treatment in sports medicine. [15, 16]

The mean NRS before treatment was 5.88 $( \pm 1.45)$, the mean NRS after treatment decreased to $3.07( \pm 1.62)$, and the p-value was 0.046 , showing statistically significant difference. NRS difference between before and after treatment according to the type of injury was $3.01( \pm 1.59)$ for muscle injury, $3.00( \pm 3.00)$ for others, and 2.43 ( \pm 2.44 ) for joint (ligament) injury. There was no difference in NRS for bone injury or laceration, but it was not statistically significant. The difference between NRS before and after treatment according to the cause of injury was $3.11( \pm 1.47)$ for aggravation or exacerbation of an existing injury, $3.00( \pm 4.24)$ for Illness, 2.82 $( \pm 1.74)$ for new injury, $2.40( \pm 2.70)$ for others, and $2.33( \pm 2.07)$ for recurrence of a previous injury that had fully resolved, but it was not statistically significant. We aimed to compare the NRS changes according to injury type or cause of injury, but the sample was insufficiently large to produce a significant result.

In this study, 37 cases (75.5\%) were highly satisfied with the treatment, 10 cases (20.4\%) sere satisfied, and 2 cases (4.1\%) were partially satisfied. All patients were foreign athletes and officials who had difficulty in receiving $\mathrm{KM}$ treatments, and, through interviews, we confirmed that most of them had never experienced $\mathrm{KM}$ treatment. Because of the high satisfaction with first-time Korean medical care, it is expected that the use of KM treatments will increase in global sports medicine. In this study, patients who had busy schedules only participated in our study for a short time, hence pain was evaluated in these patients using a simple NRS measurement, and we were not able to evaluate their functional recovery or athletic performance through physical examination. In the future, further investigations on the long-term application of KM treatment, along with various examinations, will be required to measure the effect of $\mathrm{KM}$ in sports medicine.

\section{Conclusion}

This study showed that knee and lower leg injuries (S80-S89) were the most frequent injuries in the KM clinic of the 2019 Chungju World Martial Arts Masterships. We provided KM treatments including acupuncture, cupping therapy, Chuna manual therapy, herbal medicine, dressing, and taping. Acupuncture was the most frequently performed and the most satisfactory treatment. The average NRS after treatment significantly decreased, suggesting that $\mathrm{KM}$ is effective in controlling pain in sports injuries. The overall satisfaction was also high with $\mathrm{KM}$ treatments. In order to play the important role in the treatment of sports medicine, it would be 
required the agreement on specific treatment depending on each sports injury. Additionally, further studies evaluating the effects of pain control, functional recovery, and performance improvement are required.

\section{Reference}

1. J. Filshie, A. White, M. Cummings. Medical Acupuncture: A Western Scientific Approach $2^{\text {nd }}$ edition. 2016:579-580

2. Y. H. Lee, Y. B. Park, J. K. Kim. The study of Acupuncture \& Moxibustion Hospital in 24th Seoul Olympic Games. Journal of Korean Medicine 1988;9(2):148-152.

3. M. J. Oh, S. M. Lee, J. M. Shin, J. H. Cho. The Study of Patients Visited Korean Medical Clinic in Athletes' Village Polyclinic at The 2014 Incheon Asian Paralympic. J Sports Korean Med 2016;15(2):1-6.

4. M. S. Jeon, S. Y. Bae. The Study of Athlete Patients Visited Korean Medical Clinic in CheongJu-Gym at the 2016 CheongJu World Martial Arts Masterships. J Sports Korean Med 2016;16(1):51-56.

5. J. M. Shin, M. J. Oh, J. H. Park. The Study of Patients Visited Korean Medical Clinic in Athletes' Village Hospital at The PyeongChang 2018 Paralympic Winter Games. J Sports Korean Med 2018;18(1):33-39.

6. M. J. Oh, I. M. Son, J. M. Shin, J. H. Cho. The Study of Patients Visited Korean Medical Clinic in Athletes' Village Hospital at The 28th Univesiade Gwanju Korea . J Sports Korean Med 2016;16(1):1-7.

7. The Society of Korean Medicine Rehabilitation. Korean Rehabilitation Medicine $3^{\text {rd }}$ ed. Seoul (Korea): Koonja Publishing Co; 2015:210.
8. The Korean Orthopaedic Association. Orthopedics $7^{\text {th }}$ ed. Seoul (Korea): Choisinuihaksa; 2013: 1573.

9. Korean Acupuncture \& Moxibustion Medicine Society. The acupuncture and moxibustion medicine. Seoul (Korea) : Hanmibook; 2016: 584

10. Z. P. Lin, Y. H. Chen, F. Chia, H. J. Wu, L. W. Lan, J. G. Lin. Episodes of injuries and frequent usage of traditional Chinese medicine for taiwanese elite wrestling athletes. The American Journal of Chinese Medicine, 2011;39(2):233-241.

11. C. S. Yang, E. Y. Lee, E. H. Hwang, O. J. Kwon, J. H. Lee. Management of Sport Injuries with Korean Medicine: A Survey of Korean National Volleyball Team. Evidence -Based Complementary and Alternative Medicine 2016:7

12. Z. P. Lin, L. W. Lan, T. Y. He et al. Effects of acupuncture stimulation on recovery ability of male elite basketball athletes, The American Journal of Chinese Medicine 2009;37(3):471481.

13. P. Urroz, B. Colagiuri, C. A. Smith, and B. S. Cheema. Effect of acute acupuncture treatment on exercise performance and postexercise recovery: a systematic review. Journal of Alternative and Complementary Medicine 2013;19(1):9-16.

14. G. Musumeci. Could Cupping Therapy Be Used to Improve Sports Performance?. Journal of Functional Morphology and Kinesiology 2016;1(4):373-377

15. S. S. Lee, Y. S. Lee. Review of doping control drugs in herbal medicine. Journal of Korean Medicine 1990;11(2):18-22

16. J. K. Kim, Y. S. Chun, S. K. Kang, H. C. 
(588) Journal of Korean Medicine 2019;40(4)

Cho, The Use of Herbals/Traditional Products Supplementation and Doping Test in Elite Athletes. Korean Journal of Sport Science 2009;20(4):734-742

\section{ORCID}

Jaesung Lee https://orcid.org/0000-0002-6823-4669

Jonghan Park https://orcid.org/0000-0002-7392-8243

Jangmi Park https://orcid.org/0000-0002-2211-053X

Chamkyul Lee https://orcid.org/0000-0002-0156-310X

Suhyeon Jeong https://orcid.org/0000-0003-2976-6424

Kyunghwan Kong https://orcid.org/0000-0002-2018-9926

Eunyong Lee https://orcid.org/0000-0001-5819-1602 